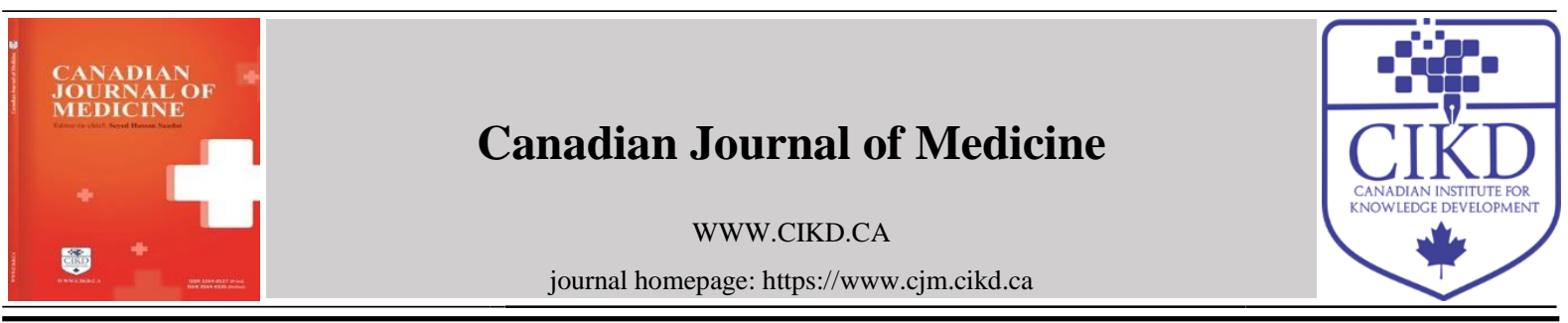

\title{
Intracholecystic Papillary-Tubular Neoplasms (ICPNs): A Pathology Report
}

\author{
Atena Azami ${ }^{1 *}$, Fahimeh Shahjoei ${ }^{2}$ \\ ${ }^{1}$ Department of Pathology, School of Medicine, Alborz University of Medical Sciences, Alborz, Iran \\ ${ }^{2}$ Emergency Department, Baqiyatallah University of Medical Sciences, Tehran, Iran
}

Keywords:

ICPN, Pathology, Colon, Surgery

\section{Received}

07 July 2021

Received in revised form

17 July 2021

Accepted

17 July 2021

*Correspondence:

azamia66@gmail.com

\begin{abstract}
We presented a 60-year-old man who underwent a colonoscopy examination of a polypoid mass with a wound surface of $1.1 \mathrm{~cm}$ in hepatic flexure. An adenocarcinoma of intestinal type was diagnosed based on the biopsy report, and patient was referred to the hospital for colectomy. In colonoscopy and biopsy, the polypoid mass was completely removed, and despite different sections of the whole specimen in the colectomy specimen, any mass was not found, while only one out of three identified lymph nodes were involved. In laboratory tests, CBC had anemia: ( $\mathrm{Hb}: 10.8 \mathrm{mg} / \mathrm{dl}$ ), elevated CEA tumor marker (range: $18 \mathrm{ng} / \mathrm{ml}$ ), and lipid profile disorder together with high cholesterol $(300 \mathrm{mg} / \mathrm{dl})$, indicating colon cancer manifestation.
\end{abstract}

CCIKD Publishing

We presented a 60-year-old man who underwent a colonoscopy examination of a polypoid mass with a wound surface of $1.1 \mathrm{~cm}$ in hepatic flexure. An adenocarcinoma of intestinal type was diagnosed based on the biopsy report, and patient was referred to the hospital for colectomy. In colonoscopy and biopsy, the polypoid mass was completely removed, and despite different sections of the whole specimen in the colectomy specimen, any mass was not found, while only one out of three identified lymph nodes were involved. 
In laboratory tests, $\mathrm{CBC}$ had anemia: (Hb: $10.8 \mathrm{mg} / \mathrm{dl}$ ), elevated CEA tumor marker (range: $18 \mathrm{ng} / \mathrm{ml})$, and lipid profile disorder together with high cholesterol $(300 \mathrm{mg} / \mathrm{dl})$, indicating colon cancer manifestation.

Simultaneous ultrasound showed a polyp in the gallbladder measuring 9 × $15 \mathrm{~mm}$, simultaneous colectomy and cholecystectomy were performed, and its diagnosis was Intracholecystic papillary-tubular neoplasm with low-grade dysplasia. And there was nothing in favor of invasion in various cuts; only a mild gallbladder dysplasia and cholesterol polyps were observed. Taken together, the diagnosis of intracholecystic papillary-tubular neoplasm (ICPN) was made for the patient

ICPN is a recently proposed pre-invasive neoplastic lesion and is rarely characterized by papillary growth in the gallbladder. ICPN is defined as gallbladder lesions of intra-ductal papillary neoplasm of the bile duct (IPNB). IPNB is a premalignant lesion of the biliary tract and is the counterpart of intra-ductal papillary-mucinous neoplasm (IPMN) in the pancreatic duct epithelium. ICPN is a papillary tumor generally detected macroscopically and is sometimes diagnosed by imaging findings

Microscopic examination showed back-to-back epithelial units in tubular and tubulepapillary configuration with limited stroma. Mixed morphologic patterns including biliary and gastric phenotypes (i.e., pyloric and foveolar types) were identified. Biliary phenotype composed of the cells had abundant eosinophilic cytoplasm, elongated oval nuclei, distinct nucleoli, and a mild degree of atypia (Figure 1A and 1B). Another component is the gastric foveolar type with elongated glands lined by tall columnar cells with pale mucinous cytoplasm (Figure 1C). Gastric pyloric phenotype with pyloric glands-like features is also identified (Figure 1D). No invasion was present in serial sections. 


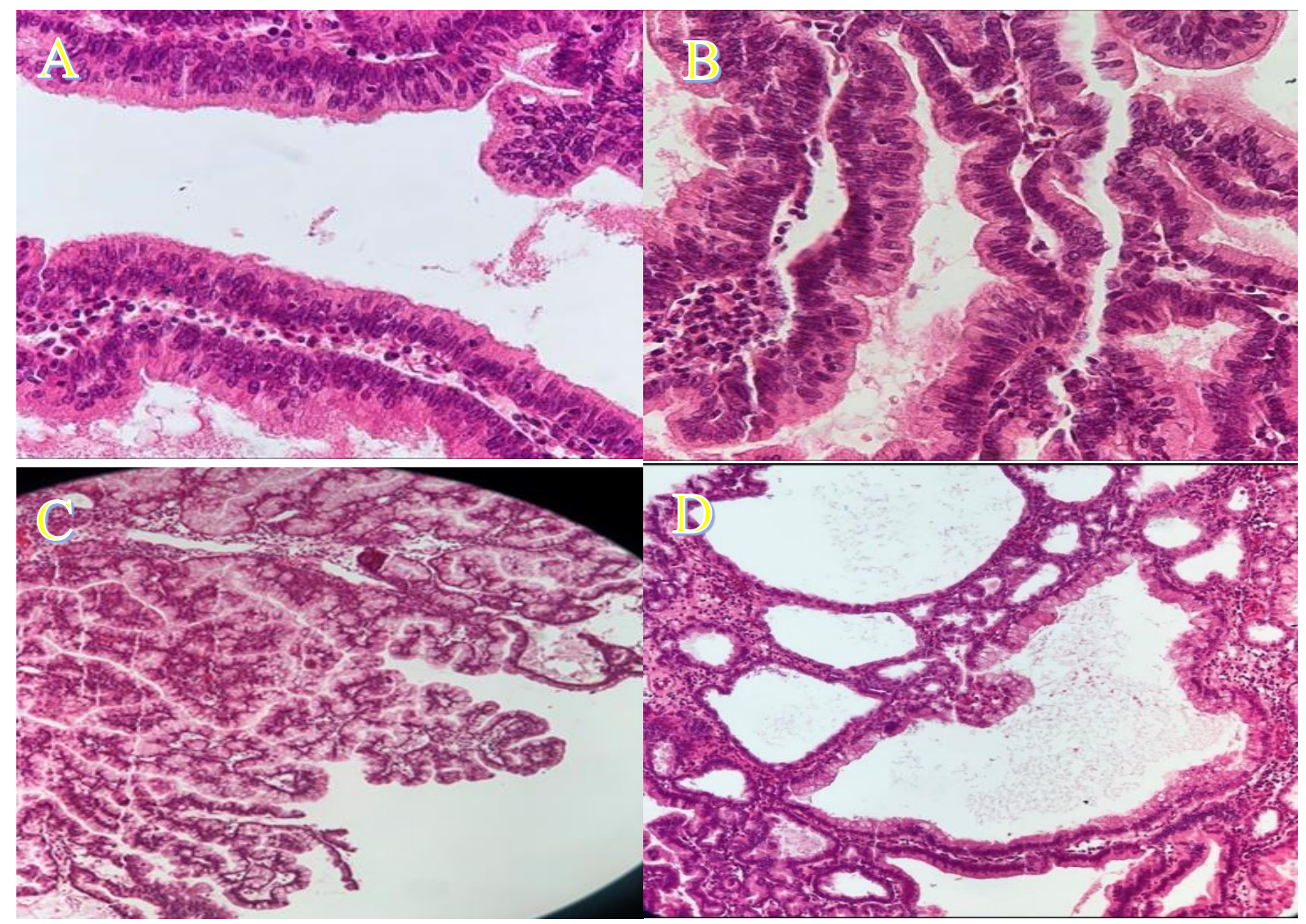

Figure 1. A: Biliary phenotype. B: Biliary phenotype with low-grade dysplasia. C: Papillary growth pattern consists of gastric foveolar phenotype. D: Gastric pyloric phenotype.

\section{Acknowledgements}

Not applicable.

\section{Disclosure Statement}

No potential conflict of interest was reported by the authors.

\section{Funding Acknowledgements}

Not applicable. 